\title{
Expression and clinical significance of tyrosine phosphatase SHP2 in thyroid carcinoma
}

\author{
ZHONG-QIAN HU ${ }^{1 *}$, RUI MA $^{2 *}$, CHI-MIN ZHANG ${ }^{1}$, JIA LI $^{1}$, \\ LING LI $^{1}$, ZHONG-TING HU ${ }^{2}$, QI GAO ${ }^{1}$ and WEI-MIN LI ${ }^{1}$ \\ ${ }^{1}$ Department of Ultrasound, Zhongda Hospital, School of Medicine, Southeast University, Nanjing, Jiangsu 210009; \\ ${ }^{2}$ Department of Cardiology, Jinling Hospital, Nanjing, Jiangsu 210002, P.R. China
}

Received August 18, 2014; Accepted May 7, 2015

DOI: $10.3892 / \mathrm{ol} .2015 .3479$

\begin{abstract}
Protein-tyrosine phosphatase SHP2 is encoded by the gene PTPN11. SHP2 is hypothesized to have a critical role in cancer, via the activation of mutations that have been detected in several types of leukaemia and in certain solid tumours, including liver, breast, gastric and cervical cancer. However, to the best of our knowledge, there have been no previous reports evaluating the significance of SHP2 expression in thyroid cancer. The present study evaluated SHP2 expression in 65 thyroid cancer specimens, 40 specimens of self-matched adjacent peritumour tissues and 40 specimens of normal thyroid tissue, using immunohistochemical and western blot analyses with an anti-SHP2 antibody. Western blotting was also used to assess SHP2 expression in thyroid cancer cell lines (SW579, IHH-4, FTC-133, TPC-1, DRO, TA-K, and ML-1) and Nthy-ori3-1 normal thyroid cells. In addition, SHP2 antisense oligonucleotides were used to block SHP2 expression in SW579 cells, and growth inhibition assays were conducted. Increased SHP2 expression was detected in the tumour tissues compared with that of the normal thyroid tissues $(\mathrm{P}<0.05)$. SHP2 expression was significantly correlated with poor tumour differentiation $(\mathrm{P}<0.05)$, late $\mathrm{TNM}$ stage $(\mathrm{P}<0.05)$ and lymph node metastasis $(\mathrm{P}<0.05)$, suggesting that SHP2 may represent a potential target for thyroid cancer therapy.
\end{abstract}

\section{Introduction}

Protein tyrosine phosphorylation is a key regulatory mechanism in eukaryotic cell physiology (1-3). The aberrant expression or function of protein tyrosine kinases (PTKs) and/or protein tyrosine phosphatases (PTPs) may result in the development

Correspondence to: Dr Zhong-Qian $\mathrm{Hu}$, Department of Ultrasound, Zhongda Hospital, School of Medicine, Southeast University, 87 Ding Jia Qiao, Nanjing, Jiangsu 210009, P.R. China E-mail: hzq920@126.com

*Contributed equally

Key words: thyroid cancer, SHP2, expression, tumour proliferation of severe human diseases, including cancer and cardiovascular disease. SHP2 is a ubiquitously expressed cytosolic tyrosine phosphatase $(4,5)$, which contains two tandem Src homology 2 (SH2) domains and a PTP domain. The SH2 domains are responsible for its role in cellular signalling. SHP2 binds to the intracellular domains of various growth factor and cytokine receptors $(6,7)$, thereby influencing cell proliferation, differentiation and migration $(8,9)$.

A considerable body of evidence supports an oncogenic role for SHP2, and since 2001, mutant and overexpressed SHP2 have been detected in patients with solid tumours, Noonan syndrome and myeloproliferative disease (1,10-12). Further research has suggested that SHP2 overexpression and gain-of-function mutations in SHP2 are present in almost all types of solid tumours and in $50 \%$ of Noonan syndrome, $35 \%$ of juvenile myelomonocytic leukaemia, $10 \%$ of myelodysplastic syndrome, $6 \%$ of B-cell acute lymphoblastic leukaemia and $3 \%$ of acute myelogenous leukaemia (10-12). SHP2 was the first identified oncogenic tyrosine phosphatase. Although PTPN11 mutations are infrequent in human solid tumours, an oncogenic role for SHP2 has been reported in several types of tumour. Evidence suggests that somatic gain-of-function mutations of the PTPN11 gene are present in certain lung adenocarcinomas $(13,14)$, breast cancer $(15-17)$, gastric cancer (18), laryngeal carcinoma (19) and oral cancer (20). These observations suggest that PTPN11/SHP2 may have a tumourigenic function. Recently, investigators have also found that SHP2 is downregulated and may exert a tumour suppressor role in hepatocellular (21) and colon (22) carcinogenesis.

The present study aimed to evaluate whether SHP2 expression is altered in thyroid cancer, and investigate the mechanisms and clinical significance of SHP2 in human thyroid cancer. SHP2 protein expression was investigated in 65 patients with thyroid cancer, and paired adjacent normal tissue samples were collected from 40 patients. Associations between protein expression, patient clinical characteristics and prognostic outcomes were also investigated.

\section{Materials and methods}

Clinical samples and human thyroid cancer cell lines. Sixty-five thyroid cancer tissues were included in the present study. Patients ages ranged from 23-70 years, with a mean age 
of 43 years. Thyroid tissue was excised, and the diagnosis was confirmed at the Department of Pathology, Zhongda Hospital (Nanjing, China). In addition, 40 normal thyroid samples were collected at the Department of Pathology for comparison. Enrolled patients had not previously been treated for thyroid cancer. Informed consent was obtained from each patient. The study design was approved by the ethics review board of the Southeast University (Nanjing, China) and patients provided written informed consent. Human thyroid cancer cells, including SW579, IHH-4, FTC-133, TPC-1, DRO, TA-K, and ML-1, and the human normal thyroid cell line Nthy-ori3-1 were obtained from the Institute of Biochemistry and Cell Biology, Chinese Academy of Sciences (Shanghai, China). All cell lines were cultured in RPMI-1640 (Gibco-BRL, Carlsbad, CA, USA) medium containing $10 \%$ foetal bovine serum (FBS; Hyclone, GE Healthcare Life Sciences, Logan, UT, USA), penicillin $(100 \mathrm{U} / \mathrm{ml})$, streptomycin $(100 \mathrm{U} / \mathrm{ml})$ and amphotericin $\mathrm{B}(0.25 \mathrm{mg} / \mathrm{ml})$ at $37^{\circ} \mathrm{C}$ with $5 \% \mathrm{CO}_{2}\left(\mathrm{GBCBIO}^{\mathrm{TM}}\right.$ Technologies, Guangzhou, China).

Immunohistochemistry (IHC). Sections $(4 \mu \mathrm{m})$ were blocked with $1 \%$ bovine serum albumin (BSA) and $0.01 \%$ Triton X-100 (Amresco LLC, Solon, OH, USA) for $1 \mathrm{~h}$ at room temperature, and then incubated with the primary monoclonal rabbit anti-human SHP2 (1:500; cat. no. 20145-1-AP; ProteinTech Group, Inc., Chicago, IL, USA) antibody overnight at $4^{\circ} \mathrm{C}$. The negative controls were obtained by omitting the primary antibody or incubating the sections with normal rabbit immunoglobulin G (Vector Laboratories Inc., Burlingame, CA, USA) or phosphate-buffered saline (PBS). Detection was performed by incubation with horseradish peroxidase-conjugated polyclonal goat anti-rabbit secondary antibody $(1: 5,000$; cat. no. A21020; Dako North America, Inc., Carpinteria, CA, USA) for $1 \mathrm{~h}$ at room temperature. The sections were incubated with the avidin-biotin-peroxidase complex (Vector Laboratories Inc; 1:100 in PBS) for $1 \mathrm{~h}$ and developed in $0.05 \%$ 3,3'-diaminobenzidine (Sigma-Aldrich, St. Louis, MO, USA) containing $0.003 \% \mathrm{H}_{2} \mathrm{O}_{2}$ in PBS.

SHP2 staining was independently evaluated by two experimenters who were blinded to the clinical and follow-up information. The IHC score was determined using the $\mathrm{H}$-score system (22), which considers the staining intensity and the percentage of cells stained at a specific range of intensity. Consistent with the known intracellular localisation of SHP2, thyroid cancer specimens with positive staining demonstrated a cytoplasmic distribution of SHP2. Using the scoring system described previously, strong cytoplasmic reactivity of $>10 \%$ of all tumour cells was designated as $3+$, moderate reactivity as $2+$ and faint reactivity as $1+$. Faint reactivity not discernible above background or the absence of staining was considered negative for SHP2 expression. A reactivity of $2+$ or $3+$ was considered positive for SHP2 expression. Microscopically-confirmed sections of normal thyroid specimens were used as the controls (22).

Western blotting. The immunoblotting procedure was conducted as described previously (16). In brief, proteins $(10 \mu \mathrm{g})$ were separated on $10 \%$ SDS-PAGE (Sigma-Aldrich) by electrophoresis. The separated proteins were transferred onto nitrocellulose membranes, and the membranes were blocked with $5 \%$ BSA or $5 \%$ nonfat milk in $0.01 \mathrm{M}$ PBS (pH 7.4) and 0.05\% PBS-Tween-20 (PBS-T; Amresco LLC) at room temperature for $1 \mathrm{~h}$. Subsequently, the membranes were probed with primary SHP2 antibody overnight at $4^{\circ} \mathrm{C}$. Following three rapid washes in PBS-T, the membranes were incubated with horseradish peroxidase-conjugated goat anti-rabbit secondary antibody (cat. no. A21020; Dako North America, Inc.). The immune complexes were detected using an enhanced chemiluminescence kit (GE Healthcare Life Sciences, Chalfont, UK). The same membranes were then stripped in stripping buffer for $15 \mathrm{~min}$ at room temperature, and re-probed with a rabbit anti-mouse actin monoclonal antibody (1:5,000; cat. no. sc-47778; Santa Cruz Biotechnology, Inc.). The images were scanned and the signal intensity was quantified with Image J software version $1.43 \mathrm{~g}$ (National Institutes of Health, Bethesda, MD, USA).

Growth inhibition assay. Sense S-oligonucleotides (Roche Diagnostics Corporation, Indianapolis, IN, USA) were synthesised to nucleotides 1-18 of the SHP2 messenger RNA (5'-ATGACATCGCGGAGATGG-3'), and antisense S-oligonucleotides were synthesised to the complementary strand (5'-CCATCTCCGCGATGTCAT-3'). One day prior to transfection, thyroid cancer cells $\left(1 \times 10^{5}\right.$ cells/well) were plated in 6-well tissue-culture plates. Thyroid cancer cells were incubated with SHP2 antisense S-oligonucleotides at various concentrations $(0,1,3$ and $5 \mu \mathrm{M})$, according to the manufacturer's instructions. Following $48 \mathrm{~h}$ of culture, the cells were collected for analysis of apoptosis using flow cytometry. The cells were washed with cold PBS and resuspended in binding buffer according to the instruction of the Annexin V-PI Apoptosis detection Kit (Beyotime Institute of Biotechnology, Shanghai, China). Fluorescein isothiocyanate (FITC)-Annexin V and propidium iodide (PI) were added to the fixed cells for $20 \mathrm{~min}$ in darkness at room temperature. Then, Annexin V binding buffer was added to the mixture prior to the measurement of fluorescence using a flow cytometer (FACSCaliber ${ }^{\mathrm{TM}}$, BD Biosciences, San Jose, CA, USA). Cell apoptosis was analyzed using CellQuest software (BD Biosciences). SHP2 expression was also analysed via western blotting with a SHP2 antibody, as described previously.

The cells were resuspended in $1 \mathrm{ml}$ complete medium containing $0.7 \%$ agar (Sigma-Aldrich) $\left(1 \times 10^{3}\right.$ cells/well in 6-well plates) and then plated on top of a bottom layer containing 1.2\% agar (Sigma-Aldrich) with complete medium. Following $24 \mathrm{~h}$, the cells were incubated with SHP2 antisense S-oligonucleotides at various concentrations $(0$ and $3 \mu \mathrm{M})$ for 4 days, with fresh medium and oligonucleotides added each day. Subsequently, the oligonucleotide-containing medium was removed, and the plates were cultured under normal conditions for 21 days, and the colonies ( $>40$ cells) were then counted and images were captured under an inverted microscope (Olympus CX31; Olympus Corporation, Tokyo, Japan). The results are expressed as the mean percentage of clonal growth in the plates. Each experiment was performed at least three times.

Statistical analysis. All experiments were performed at least three times, and all data are presented as the mean \pm standard deviation. Groups were compared by one-way analysis of variance, followed by Pearson's coefficient analysis for bivariate 
Table I. SHP2 expression in normal, peritumour and thyroid cancer tissues.

\begin{tabular}{|c|c|c|c|c|c|c|}
\hline \multirow[b]{2}{*}{ Pathological diagnosis } & \multirow[b]{2}{*}{ Cases, $\mathrm{n}$} & \multicolumn{4}{|c|}{ SHP2 expression, $\mathrm{n}$} & \multirow[b]{2}{*}{ Positive rate, $\%$} \\
\hline & & - & + & ++ & +++ & \\
\hline Thyroid cancer & 65 & 3 & 7 & 34 & 21 & 84.6 \\
\hline Peritumour & 40 & 20 & 19 & 1 & 0 & 2.5 \\
\hline Normal & 40 & 33 & 6 & 0 & 0 & 0.0 \\
\hline
\end{tabular}

Table II. Correlations between SHP2 expression in human thyroid cancer and clinicopathological parameters.

\begin{tabular}{|c|c|c|c|c|}
\hline Parameter & Cases, $\mathrm{n}$ & SHP2 overexpression, $\mathrm{n}$ & $\chi^{2}$ & P-value \\
\hline Age, years & & & 0.64 & $>0.05$ \\
\hline$<40$ & 36 & 29 & & \\
\hline$\geq 40$ & 29 & 22 & & \\
\hline Gender & & & 0.71 & $>0.05$ \\
\hline Male & 18 & 14 & & \\
\hline Female & 47 & 37 & & \\
\hline Tumour, $\mathrm{cm}$ & & & 0.48 & $>0.05$ \\
\hline$<1$ & 12 & 9 & & \\
\hline $1-4$ & 45 & 35 & & \\
\hline$>4$ & 8 & 7 & & \\
\hline Pathological type & & & 0.56 & $>0.05$ \\
\hline Papillary thyroid carcinoma & 31 & 24 & & \\
\hline Follicle carcinoma & 22 & 17 & & \\
\hline Medullary carcinoma & 11 & 9 & & \\
\hline Undifferentiated carcinoma & 1 & 1 & & \\
\hline TNM stage & & & 6.39 & $<0.01$ \\
\hline I-II & 39 & 27 & & \\
\hline III & 26 & 24 & & \\
\hline Differentiation grade & & & 7.08 & $<0.01$ \\
\hline I & 20 & 11 & & \\
\hline II & 26 & 21 & & \\
\hline III & 19 & 19 & & \\
\hline Lymph node metastasis & & & 8.17 & $<0.01$ \\
\hline Present & 23 & 22 & & \\
\hline Absent & 42 & 29 & & \\
\hline Capsular invasion & & & 9.25 & $<0.01$ \\
\hline Present & 20 & 19 & & \\
\hline Absent & 45 & 32 & & \\
\hline
\end{tabular}

correlation. A two-tailed probability value $\mathrm{P} \leq 0.05$ was considered to indicate a statistically significant difference.

\section{Results}

SHP2 expression is increased in thyroid cancer patient samples and cell lines. SHP2 expression was detected in thyroid cancer cells, including SW579, IHH-4, FTC-133, TPC-1, DRO, TA-K, and ML-1 cells, and the normal human thyroid cell line Nthy-ori3-1 by western blotting. Significantly enhanced SHP2 expression was observed in the thyroid cancer cells compared with that of the normal thyroid epithelium (Fig. 1A and B). In addition, SHP2 expression was evaluated in human thyroid cancer samples. IHC analysis revealed that SHP2 protein was expressed in the cytoplasm and nuclei (Fig. 1C). Further measurements using the H-score system revealed that SHP2 expression in tumour tissues was significantly higher than that in paired normal tissues $(\mathrm{P}<0.001$; Fig. $1 \mathrm{C})$. No significant difference was observed between SHP2 expression in the normal thyroid and peritumour tissues $(\mathrm{P}=0.8994$; Table I). 
A
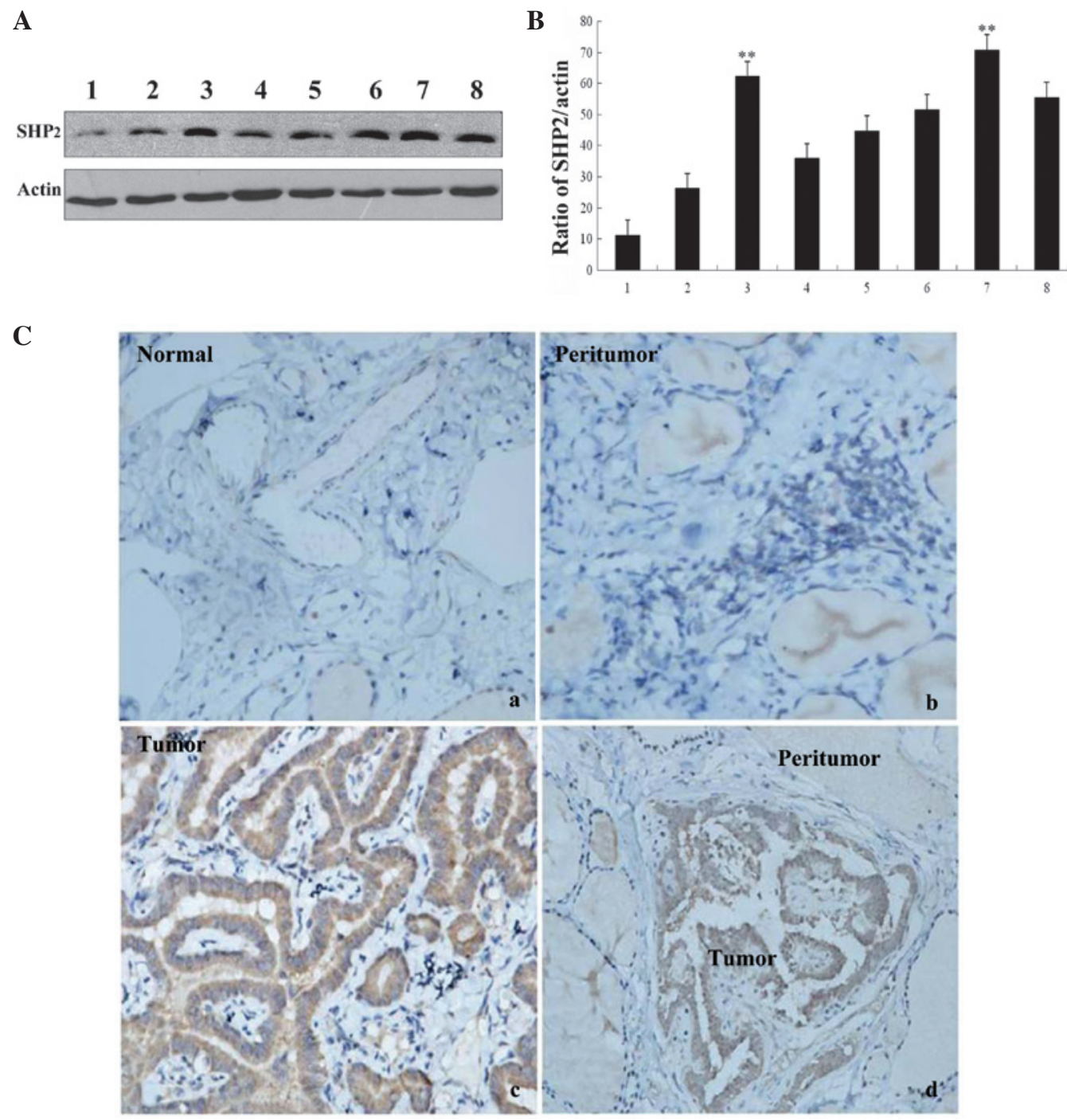

D

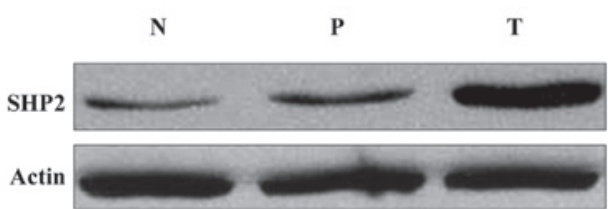

E

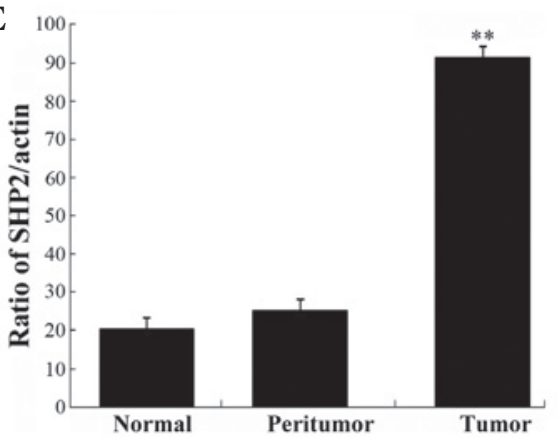

Figure 1. SHP2 is overexpressed in human thyroid cancer cell lines and tumour tissues. (A) Expression of SHP2 in normal human thyroid and thyroid cancer cell lines. Lanes: 1, Mthy-ori3-1; 2, IHH-4; 3, DRO; 4, TPC-1; 5, FTC-133; 6, TA-K; 7, SW579 and 8, ML-1. (B) Quantitative relative band intensities of the western blot presented in $\mathrm{A} .{ }^{*} \mathrm{P}<0.05$ and ${ }^{* *} \mathrm{P}<0.01$ vs. normal human thyroid cells (lane 1). (C) Representative immunohistochemical photomicrographs of human thyroid tissues (a-c: Magnification, x400, d: Magnification, x200). Increased SHP2 expression was detected in human thyroid cancers by immunohistochemical staining of 65 human thyroid cancer samples (3+), 40 self-paired peritumour tissues (1+) and 40 normal tissues (1+). (D) Western blot analysis of SHP2 protein expression in human thyroid specimens (N, normal; P, peritumour; T, tumour). (E) Semi-quantitative western blotting of human thyroid samples revealed significantly increased SHP2 protein levels in tumour tissues compared with those of normal and peritumour tissues. Actin was used as an internal control. ${ }^{* *} \mathrm{P}<001$ vs. normal tissue.

Similar results were obtained from western blot analysis (Fig. 1D and E). In conclusion, SHP2 protein expression was significantly increased in human thyroid carcinoma.

SHP2 expression is positively correlated with the degree of tumour differentiation and TNM stage. All thyroid cancer specimens were subjected to IHC analysis, and staining intensity was measured by the H-score system. SHP2 expression did not differ between genders. High SHP2 expression was significantly correlated with tumour differentiation, high TNM stage and lymph node metastasis $(\mathrm{P}<0.05$; Table II). Therefore, SHP2 may have a positive role in tumour initiation and progression. 
A
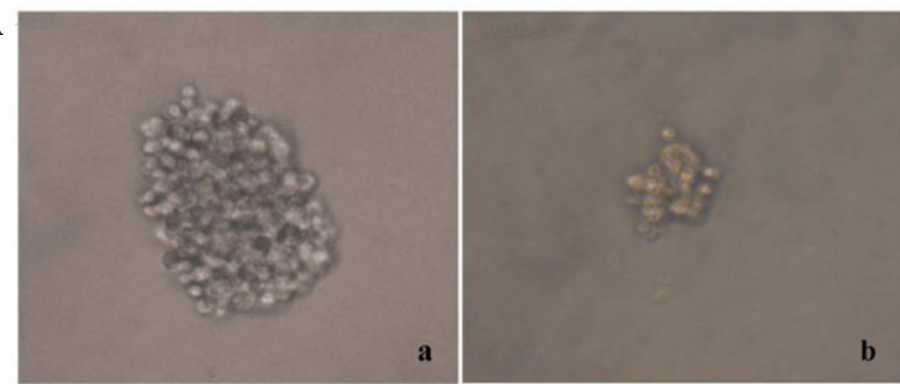

B

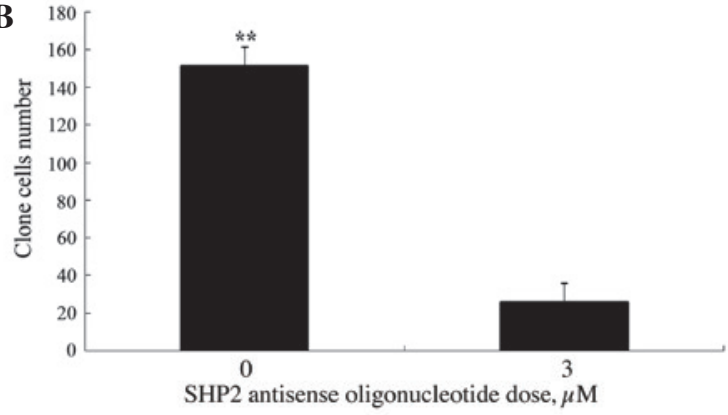

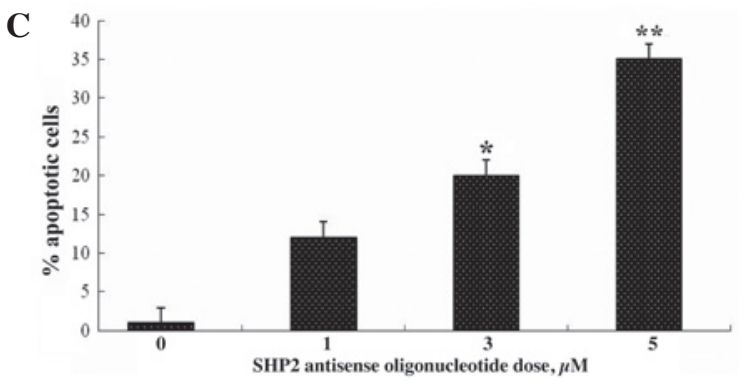

D

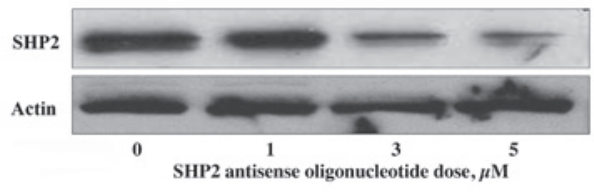

E

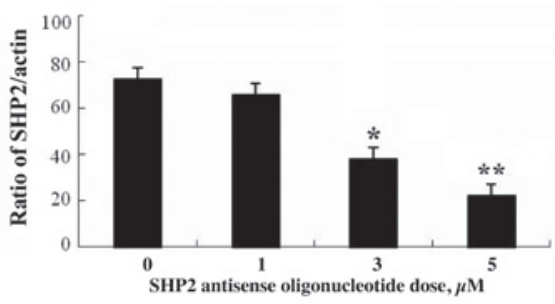

Figure 2. SHP2 antisense oligonucleotides induce growth inhibition and apoptosis of thyroid cancer SW579 cells. SW579 cells were plated in 6-well culture plates in $0.7 \%$ soft agar with 0 or $3 \mu \mathrm{M}$ SHP2 antisense oligonucleotides for 21 days. Subsequently, colonies ( $>40$ cells) were counted using an inverted microscope. (A) Microphotographs of thyroid colonies following exposure to antisense oligonucleotides at (a) $0 \mu \mathrm{M}$ (magnification, $\mathrm{x} 400$ ) and (b) $3 \mu \mathrm{M}$ (magnification, $\mathrm{x} 400$ ) for 4 days. (B) SHP2 antisense oligonucleotide exposure decreased the plate colony formation rate of SW579 cells. ${ }^{* *} \mathrm{P}<0.01$ vs. $0 \mu \mathrm{M}$. (C-E) Thyroid cancer cells were cultured in Dulbecco's modified Eagle's medium with $10 \%$ serum, and treated with various doses $(0,1,3$ and $5 \mu \mathrm{M})$ of SHP2 antisense oligonucleotides for 4 days prior to collection for analysis of (C) apoptosis with flow cytometry and (D and E) SHP2 expression by western blotting. ${ }^{*} \mathrm{P}<0.05 ;{ }^{* *} \mathrm{P}<0.01$ vs. $0 \mu \mathrm{M}$.

Suppression of SHP2 expression induces apoptosis and inhibits growth of thyroid cancer cells. To evaluate the role of SHP2 in the proliferation of thyroid cancer cells, SHP2 expression was blocked with SHP2 antisense oligonucleotides and the effects on thyroid cancer cell clone growth were evaluated. SW579 thyroid cancer cells, which express high levels of SHP2 protein, were treated with SHP2 antisense oligonucleotides at various concentrations. As shown in Fig. 2A and B, the growth of thyroid cancer cell clones was markedly suppressed following exposure to SHP2 antisense oligonucleotides for 4 days. In addition, enhanced apoptosis of thyroid cancer cells was observed, accompanied by decreased SHP2 protein expression, following SHP2 antisense treatment (Fig. 2C-E), demonstrating a positive role for SHP2 in thyroid cancer cell survival and proliferation.

\section{Discussion}

With more in-depth research regarding tumour molecular biology, the molecular mechanisms of malignancy and the process of tumour evolution in recent years, it is well understood that cancer is a disease characterised by abnormal cellular proliferation and differentiation $(23,24)$. SHP2 is a member of the PTP family and has been shown to be ubiquitously expressed in mammalian tissues. PTPs regulate several cellular processes, including cell growth, cellular differentiation, mitotic cycles and oncogenic transformation $(8,9)$. PTPs also regulate the phosphorylation state of numerous signalling molecules with PTKs, including the MAP kinase family and signal transducer and activator of transcription family proteins $(25,26)$. In recent years, there has been expanding interest in the role of SHP2 in the genesis, development and prognosis of thyroid cancer.

To date, studies have suggested that SHP2 activation may be a critical step in the initiation and continued development of multiple types of malignant tumour $(27,28)$. However, studies evaluating the expression of SHP2 in thyroid cancer are scarce. To the best of our knowledge, the results of the present study demonstrate, for the first time, that SHP2 expression is enhanced in thyroid carcinoma tissues, compared with that in normal thyroid and paracancerous tissues. SHP2 expression was demonstrated to be associated with the metastasis of thyroid cancer, suggesting its involvement in the initiation and development of thyroid cancer, and indicating that SHP2 may be useful for evaluating prognostic outcome. Based on the findings of the present study, it was speculated that SHP2 may exist in a relatively stable state in normal thyroid tissue 
and that its expression is increased during the development of lesions, to maintain cellular homeostasis and facilitate adjustment to the disorder. As malignant tumours form, SHP2 is further activated, and the overexpression of SHP2 promotes cell proliferation, growth and metastasis. The association between SHP2 protein expression and the clinicopathological parameters of thyroid carcinoma were analysed, and the results revealed that positive expression of SHP2 and cancer staging, histological differentiation, lymph node metastasis, tumour size and capsule invasion were closely associated $(\mathrm{P}<0.01)$; while patient age and gender were not significantly associated with SHP2 expression $(\mathrm{P}>0.05)$. Increased SHP2 expression is not only involved in the initiation of thyroid cancer but also has roles in invasion and metastasis, as well as tumour evolution. In addition, the results of the present study suggest that SHP2 may be a novel marker for predicting the malignant biological behaviour of thyroid cancer. Finally, SHP2 may represent a novel therapeutic target for the treatment of thyroid cancer. Such a therapy may potentially promote tumour cell apoptosis, thus inhibiting cancer cell growth and metastasis, and it may delay tumour recurrence and improve the overall survival rate of patients with thyroid cancer.

\section{Acknowledgements}

The present study was supported by the Fundamental Research Funds for the Central Universities (2242014K40004).

\section{References}

1. Grossmann KS, Rosário M, Birchmeier C and Birchmeier W: The tyrosine phosphatase SHP2 in development and cancer. Adv Cancer Res 106: 53-89, 2010.

2. Ferrari E, Tinti M, Costa S, et al: Identification of new substrates of the protein-tyrosine phosphatase PTP1B by Bayesian integration of proteome evidence. J Biol Chem 286: 4173-4185, 2011

3. Araki T, Chan G, Newbigging S, et al: Noonan syndrome cardiac defects are caused by PTPN11 acting in endocardium to enhance endocardial-mesenchymal transformation. Proc Natl Acad Sci USA 106: 4736-4741, 2009.

4. Hagihara K, Zhang EE, Ke YH, et al: Shp2 acts downstream of SDF-1alpha/CXCR4 in guiding granule cell migration during cerebellar development. Dev Biol 334: 276-284, 2009.

5. Niihori T, Aoki Y, Ohashi H, et al: Functional analysis of PTPN11/SHP-2 mutants identified in Noonan syndrome and childhood leukemia. J Hum Gene 50: 192-202, 2005.

6. Ogata $T$ and Yoshida R: PTPN11 mutations and genotype-phenotype correlations in Noonan and LEOPARD syndromes. Pediatr Endocrinol Rev 2: 669-674, 2005.

7. Oh ES, Gu H, Saxton TM, et al: Regulation of early events in integrin signaling by protein tyrosine phosphatase SHP-2. Mol Cell Biol 19: 3205-3215, 1999.

8. Bowen ME, Ayturk UM, Kurek KC, et al: SHP2 regulates chondrocyte terminal differentiation, growth plate architecture and skeletal cell fates. PLoS Genet 10: e1004364, 2014.

9. Hartman ZR, Schaller MD and Agazie YM: The tyrosine phosphatase SHP2 regulates focal adhesion kinase to promote EGF-induced lamellipodia persistence and cell migration. Mol Cancer Res 11: 651-664, 2013.
10. Qiu W, Wang X, Romanov V, et al: Structural insights into Noonan/LEOPARD syndrome-related mutants of protein-tyrosine phosphatase SHP2 (PTPN11). BMC Struct Biol 14: 10, 2014

11. Oishi K, Zhang H, Gault WJ, et al: Phosphatase-defective LEOPARD syndrome mutations in PTPN11 gene have gain-of-function effects during Drosophila development. Hum Mol Genet 18: 193-201, 2009.

12. Chan G, Kalaitzidis D, Usenko T, et al: Leukemogenic Ptpn11 causes fatal myeloproliferative disorder via cell-autonomous effects on multiple stages of hematopoiesis. Blood 113: 4414-4424, 2009.

13. Schneeberger VE, Luetteke N, Ren Y, et al: SHP2E76K mutant promotes lung tumorigenesis in transgenic mice. Carcinogenesis 35: 1717-1725, 2014.

14. Furcht CM, Muñoz Rojas AR, Nihalani D and Lazzara MJ: Diminished functional role and altered localization of SHP2 in non small cell lung cancer cells with EGFR-activating mutations. Oncogene 32: 2346-2355, 2013.

15. Sausgruber N, Coissieux MM, Britschgi A, et al: Tyrosine phosphatase SHP2 increases cell motility in triple-negative breast cancer through the activation of SRC-family kinases. Oncogene 34: 2272-2278, 2015.

16. Hu Z, Fang H, Wang X, et al: Overexpression of SHP2 tyrosine phosphatase promotes the tumorigenesis of breast carcinoma. Oncol Rep 32: 205-212, 2014.

17. Aceto N, Sausgruber N, Brinkhaus H, et al: Tyrosine phosphatase SHP2 promotes breast cancer progression and maintains tumor-initiating cells via activation of key transcription factors and a positive feedback signaling loop. Nat Med 18: 529-537, 2012.

18. Jiang J, Jin MS, Kong F, et al: Increased expression of tyrosine phosphatase SHP-2 in Helicobacter pylori-infected gastric cancer. World J Gastroenterol 19: 575-580, 2013.

19. Dong LB, Li GQ, Tian ZH, et al: Expressions of Src homology 2 domain-containing phosphatase and its clinical significance in laryngeal carcinoma. Genet Mol Res 12: 4207-4212, 2013.

20. Wang HC, Chiang WF, Huang HH, et al: Src-homology 2 domain-containing tyrosine phosphatase 2 promotes oral cancer invasion and metastasis. BMC Cancer 14: 442, 2014.

21. Bard-Chapeau EA, Li S, Ding J, et al: PTPN11/SHP2 acts as a tumor suppressor in hepatocellular carcinogenesis. Cancer Cell 19: 629-639, 2011.

22. Cai P, Guo W, Yuan H, et al: Expression and clinical significance of tyrosine phosphatase SHP-2 in colon cancer. Biomed Pharmacother 68: 285-290, 2014.

23. Sharma N, Everingham S, Ramdas B, et al: SHP2 phosphatase promotes mast cell chemotaxis toward stem cell factor via enhancing activation of the Lyn/Vav/Rac signaling axis. J Immunol 192: 4859-4866, 2014.

24. Princen F, Bard E, Sheikh F, et al: Deletion of Shp2 tyrosine phosphatase in muscle leads to dilated cardiomyopathy, insulin resistance and premature death. Mol Cell Biol 29: 378-388, 2009

25. Edwards JJ, Martinelli S, Pannone L, et al: A PTPN11 allele encoding a catalytically impaired SHP2 protein in a patient with a Noonan syndrome phenotype. Am J Med Genet A 164A: 2351-2355, 2014.

26. Müller PJ, Rigbolt KT, Paterok D, et al: Protein tyrosine phosphatase SHP2/PTPN11 mistargeting as a consequence of SH2-domain point mutations associated with Noonan Syndrome and leukemia. J Proteomics 84: 132-147, 2013.

27. Luo H, Tang C, Yang X and Zhou X: The tyrosine phosphatase SHP2: A key molecule linked both type 2 diabetes and cancers? Med Chem 4: 435-438, 2014.

28. Meng F, Zhao X and Zhang S: SHP-2 phosphatase promotes cervical cancer cell proliferation through inhibiting interferon- $\beta$ production. J Obstet Gynaecol Res 39: 272-279, 2013. 